\title{
Inhibitory effect of kaempferol on skin fibrosis in systemic sclerosis by the suppression of oxidative stress
}

\author{
OAkiko Sekiguchi 1, Chisako Fujiwara1, Sahori Yamazaki', Yuta Inoue1, Akihiko \\ Uchiyama1, Ryoko Akai², Takao Iwawaki², Osamu Ishikawa1, Sei-ichiro Motegi ${ }^{1}$
}

1. Department of Dermatology, Gunma University Graduate School of Medicine, Maebashi, Gunma, Japan

2. Division of Cell Medicine, Department of Life Science, Medical Research Institute, Kanazawa Medical University, Ishikawa, Japan

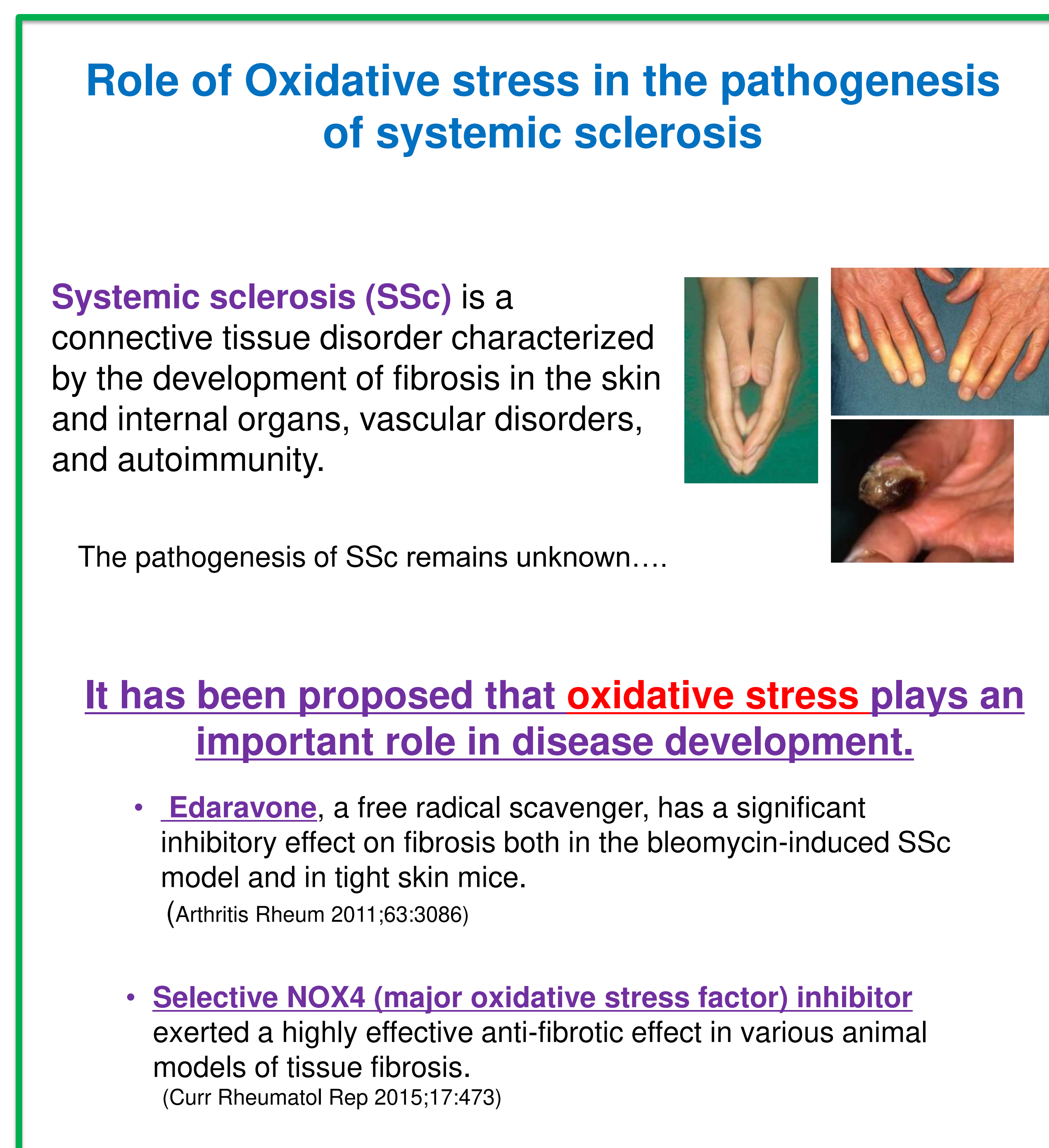

\section{Kaempferol}

Natural flavonol widely found in various vegetables, which has been reported to have excellent anti-oxidant activity.

kaempferol have a wide range of beneficial pharmacological activities.

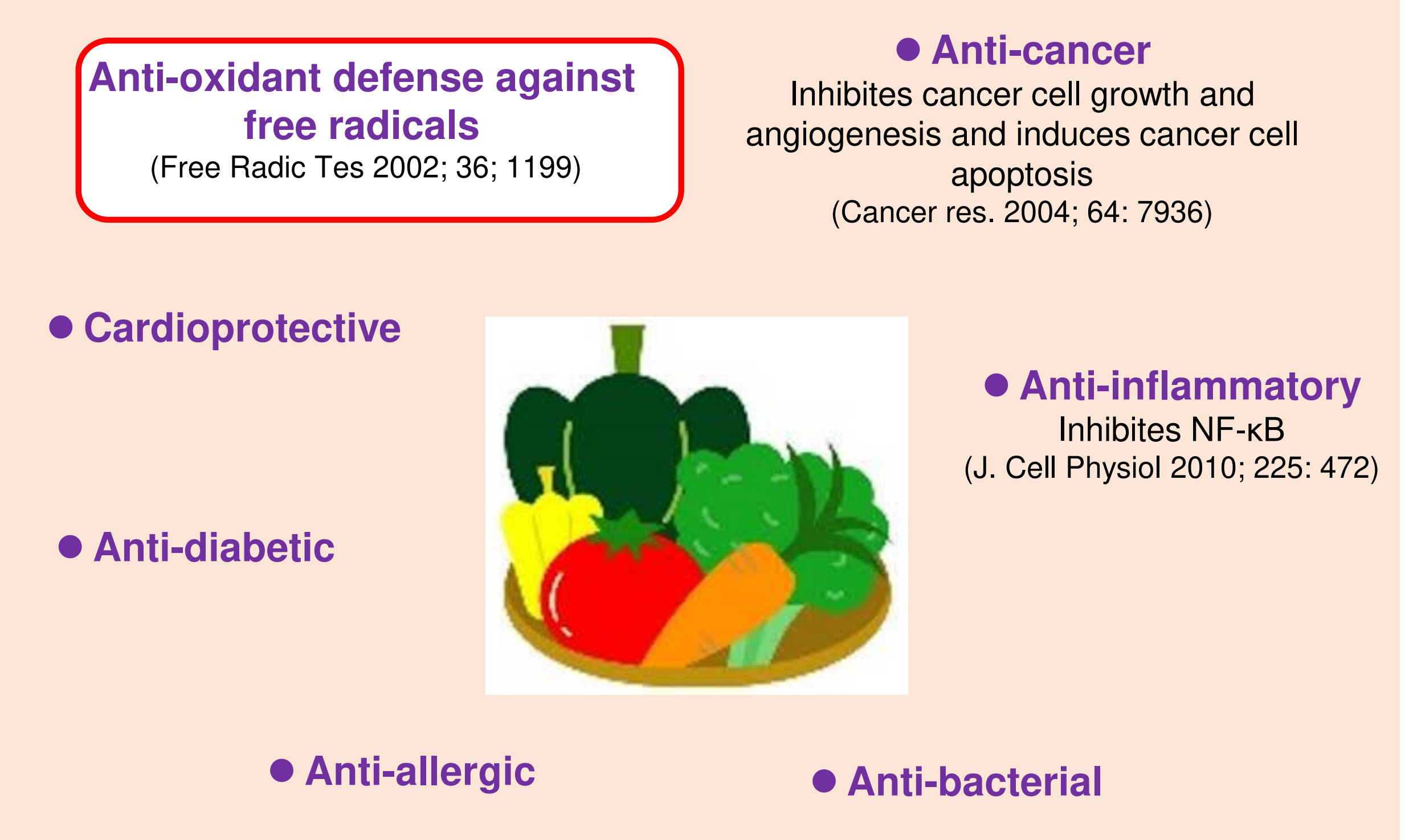

Hypothesis

Kaempferol might improve skin fibrosis in SSc patients by reducing oxidative stress?

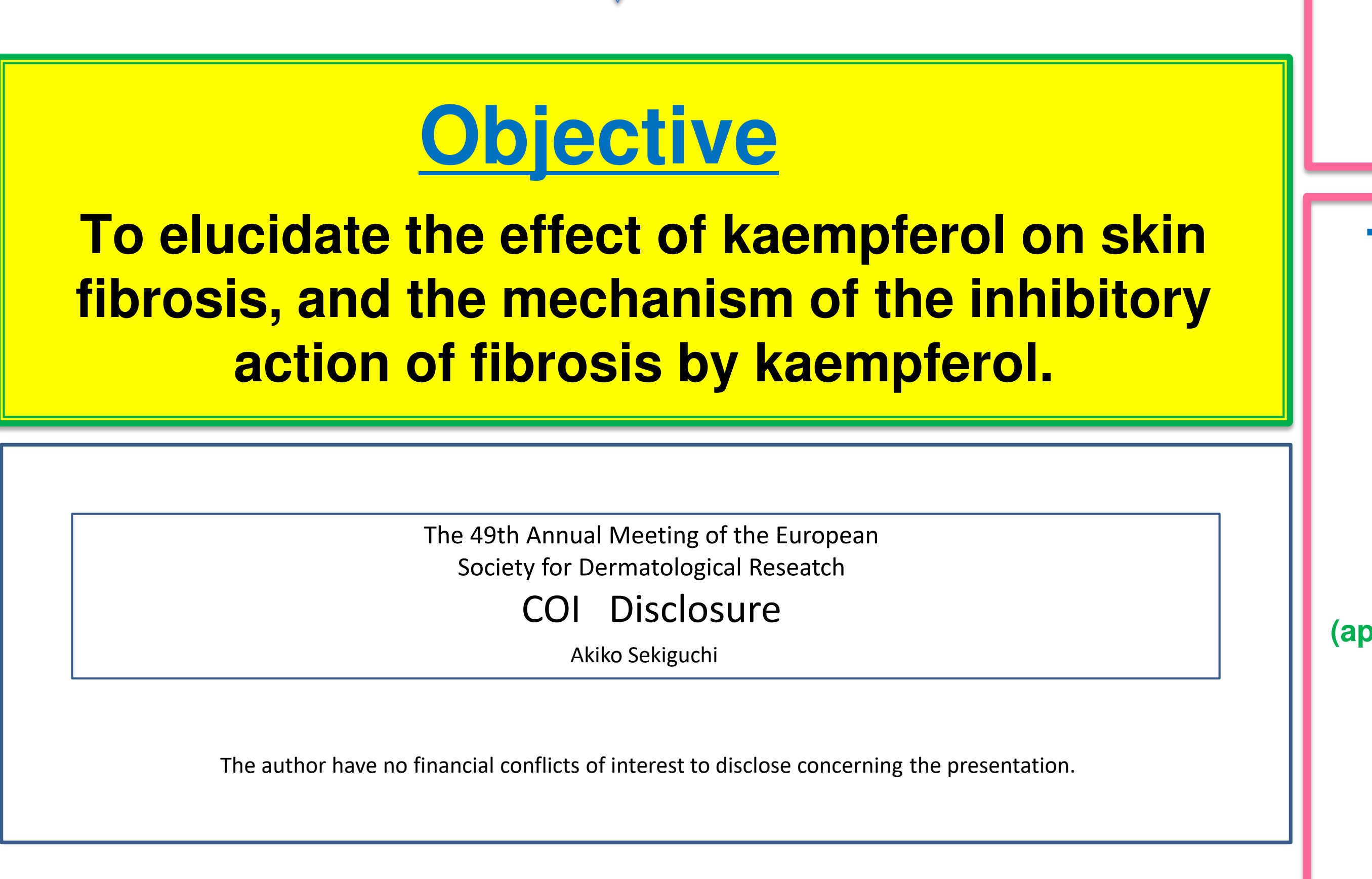

Sekiguchi A, Motegi S, Fujiwara C, Yamazaki S, Inoue U, Uchiyama A, Akai R, Iwawaki T, Ishikawa O.

Inhibitory effect of kaempferol on skin fibrosis in systemic sclerosis by the suppression of oxidative stress.

Journal of Dermatological Science. 2019 Aug 16. [Epub ahead of print].
The effect of kaemphferol on bleomycin-induced skin fibrosis in mice

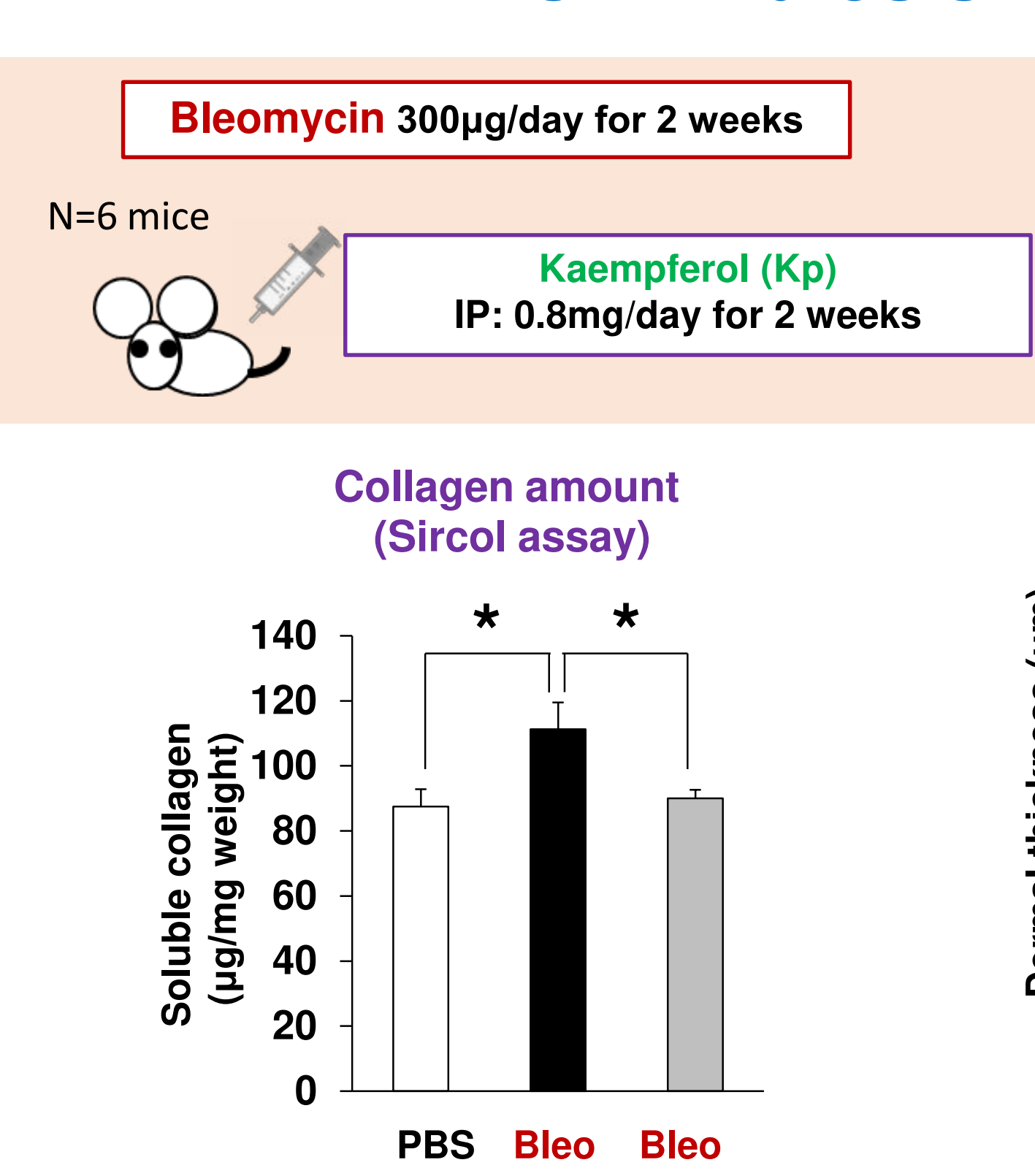

PBS Bleo Bleo

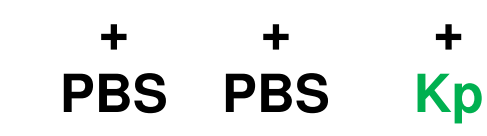

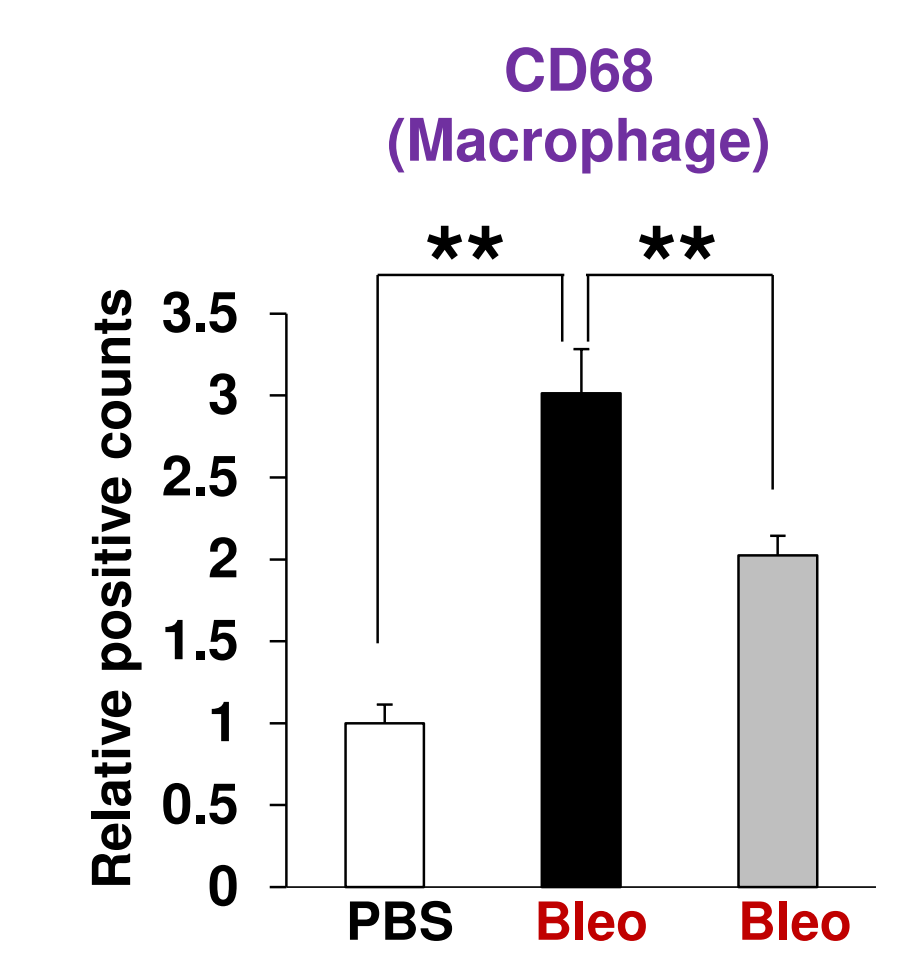

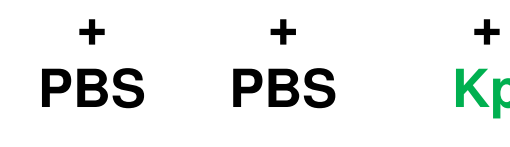

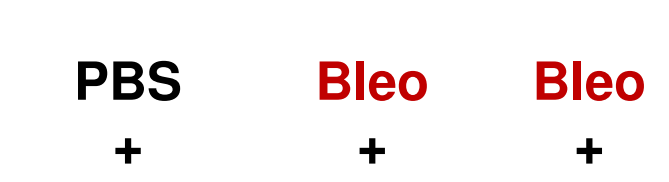

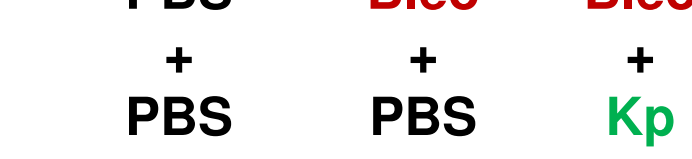

CD3
( $\mathrm{T}$ cell)

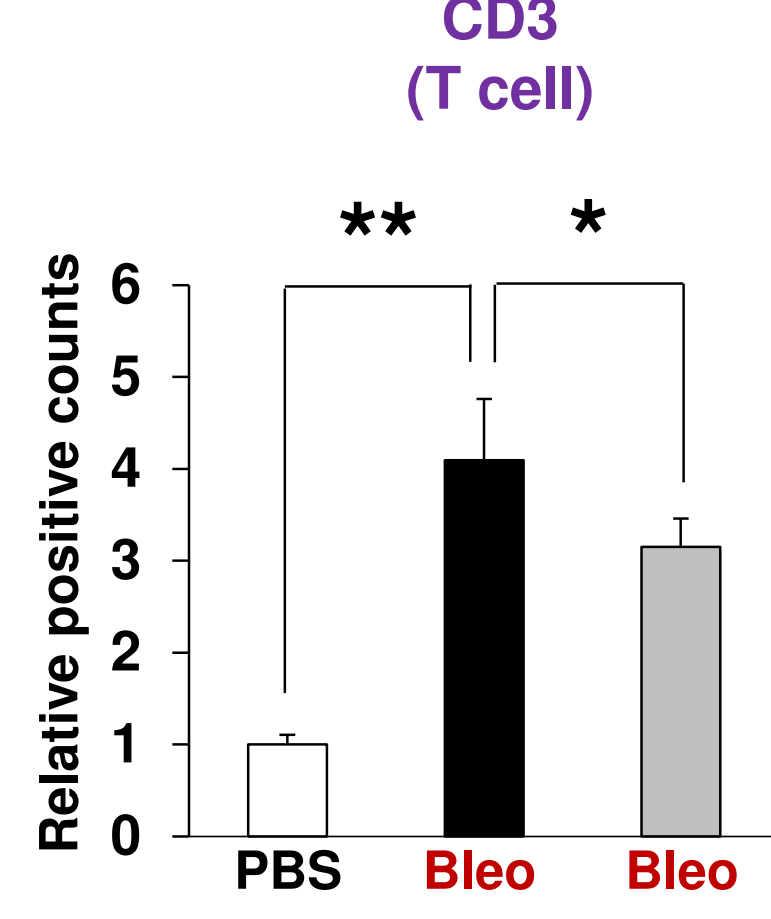

$\begin{array}{ccc}+ & + & + \\ \text { PBS } & \text { PBS } & + \\ \text { Kp }\end{array}$

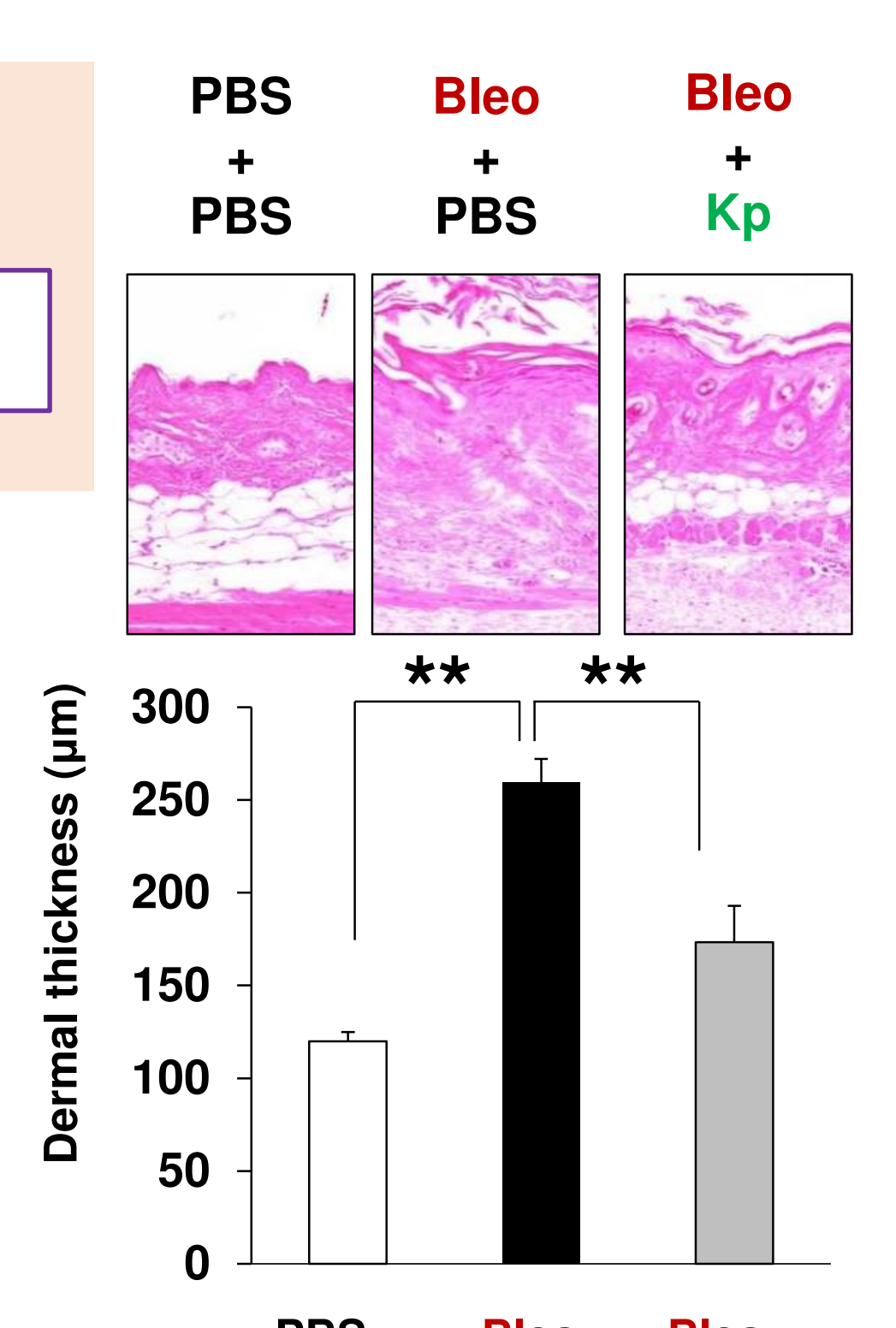

- Bleomycin induced dermal thickness inhibited by kaempherol.

The collagen amount were increased by bleomycin and decreased by kaempferol treatment.

The number of $\mathrm{CD} 68^{+}$macropheges and $\mathrm{CD} 3^{+}$Tcells in the skin were increased by bleomycin and reduced by kaempferol treatment.

Kaempferol might have inhibitory regulation of skin fibrosis and inflammatory activity.

Investigating oxidative stress in bleomycin-induced skin fibrosis using OKD 48 transgenic mice

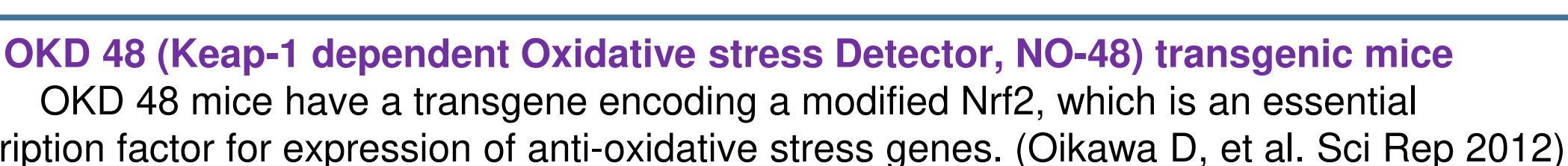

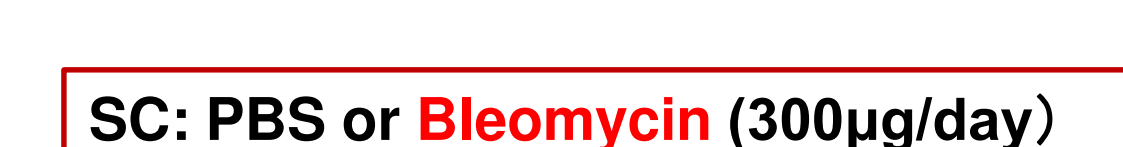

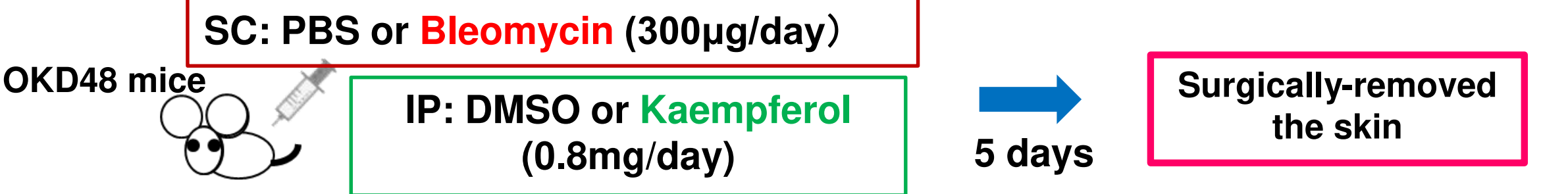

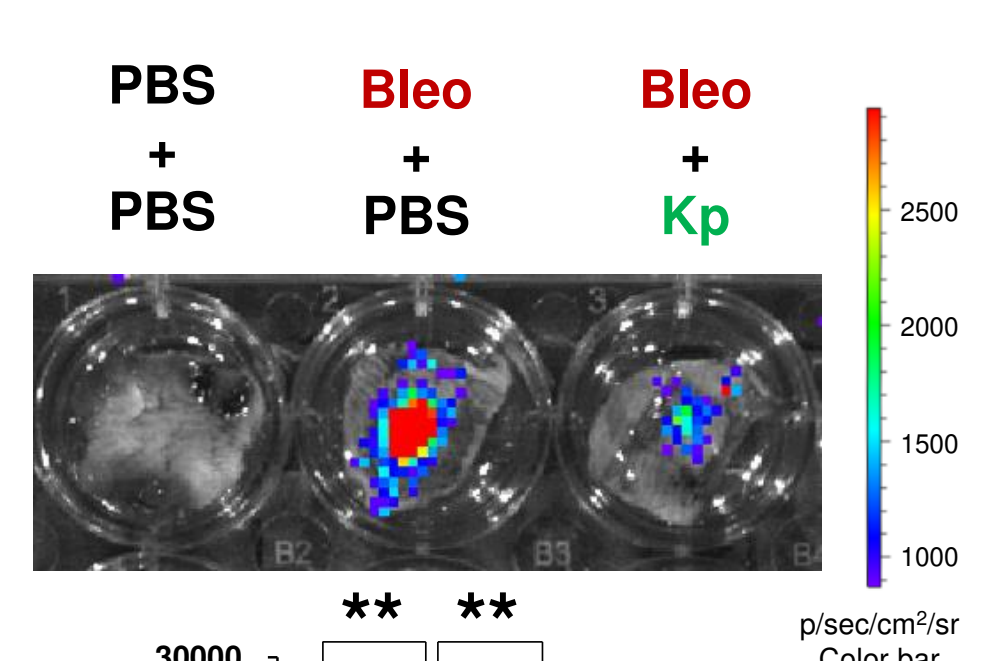

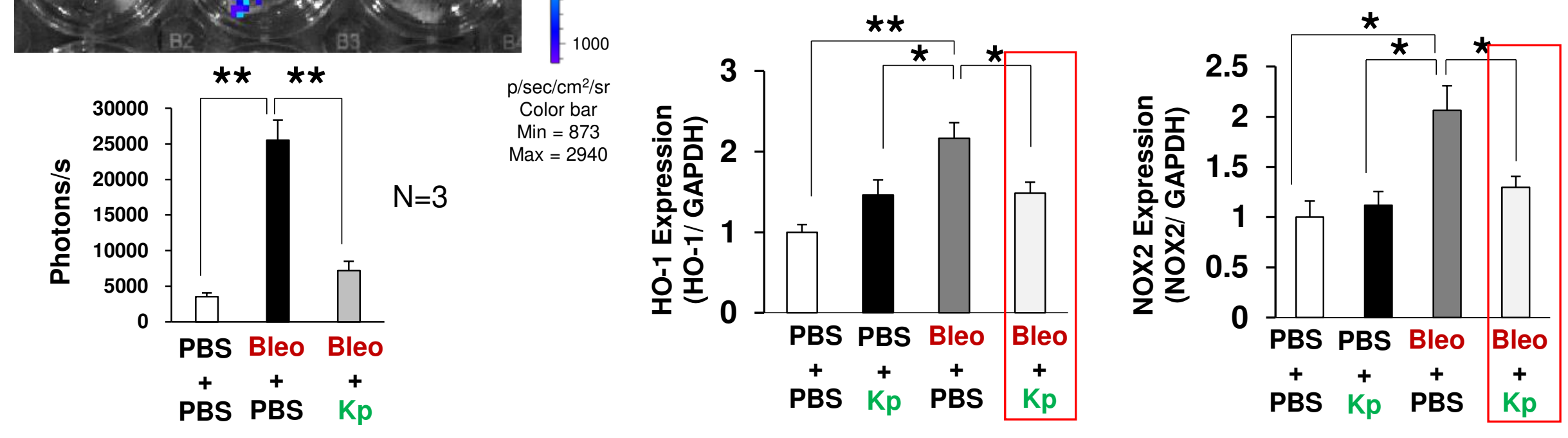

Kaempferol reduced bleomycin-induced oxidative stress in vivo.

The effect of kaempferol on bleomycin-induced apoptosis in vivo

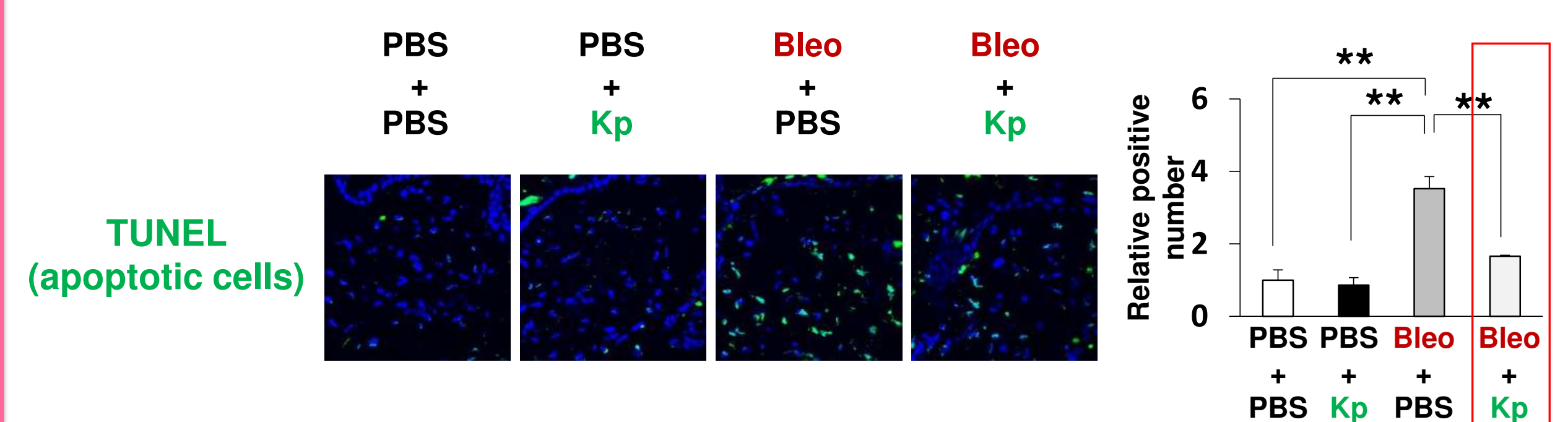

The number of apoptotic cells was increased by bleomycin bu Kaemp Kp

Investigating the effect of kaempferol on H2O2-induced ROS accumulation in vitro
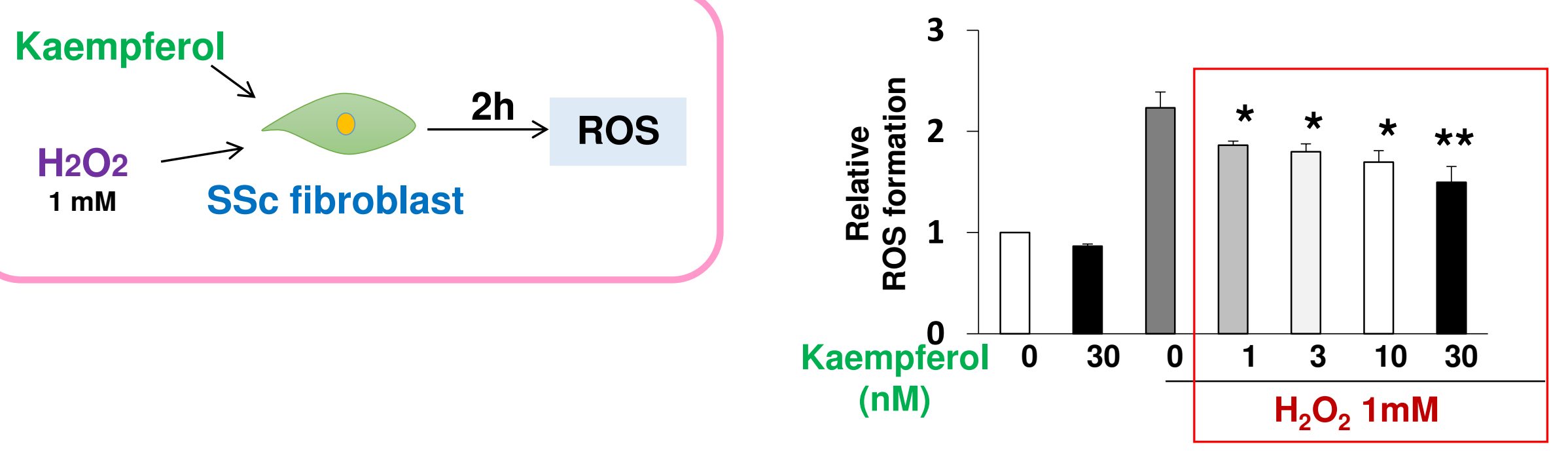

The ROS accumulation induced by bleomycin was inhibited by

kaempferol at various concentrations.

kaempferol might reduce oxidative stress and oxidative stress-induced apoptosis in vivo and vitro.

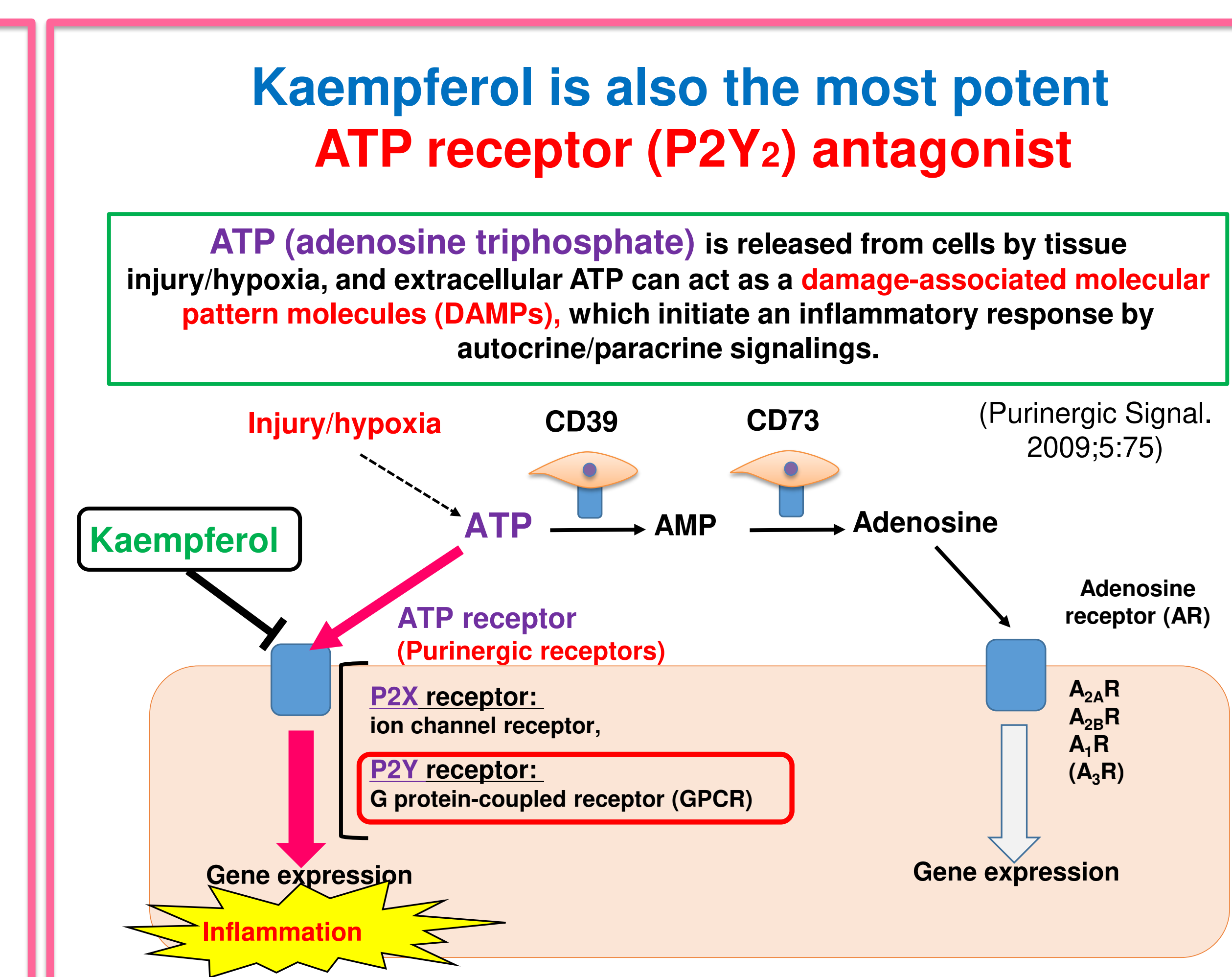

- ATP binds to purinergic receptors, such as P2X and P2Y receptors, and induce gene expression.

- Kaempferol inhibits the P2Y2 receptor.

Next

We analized the effect of the blockade of ATP/purinergic receptor signal by Kaempferol on bleomycin-induced skin fibrosis.

Kaempferol might inhibit ATP-induced IL-6 production mediated by $\mathrm{P}_{2} \mathrm{Y}_{2}$ receptor in vivo and vitro

The amount of ATP in bleomycin- ATP-induced IL-6 expression in bleomycin-

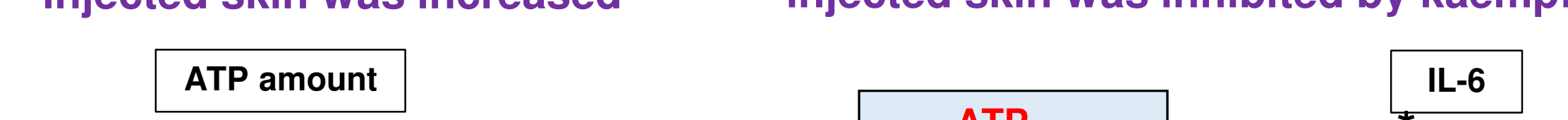

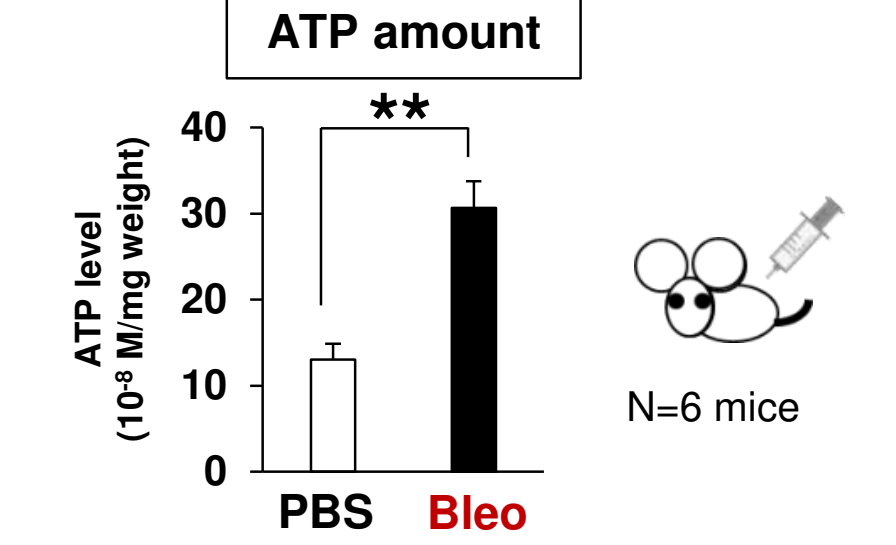

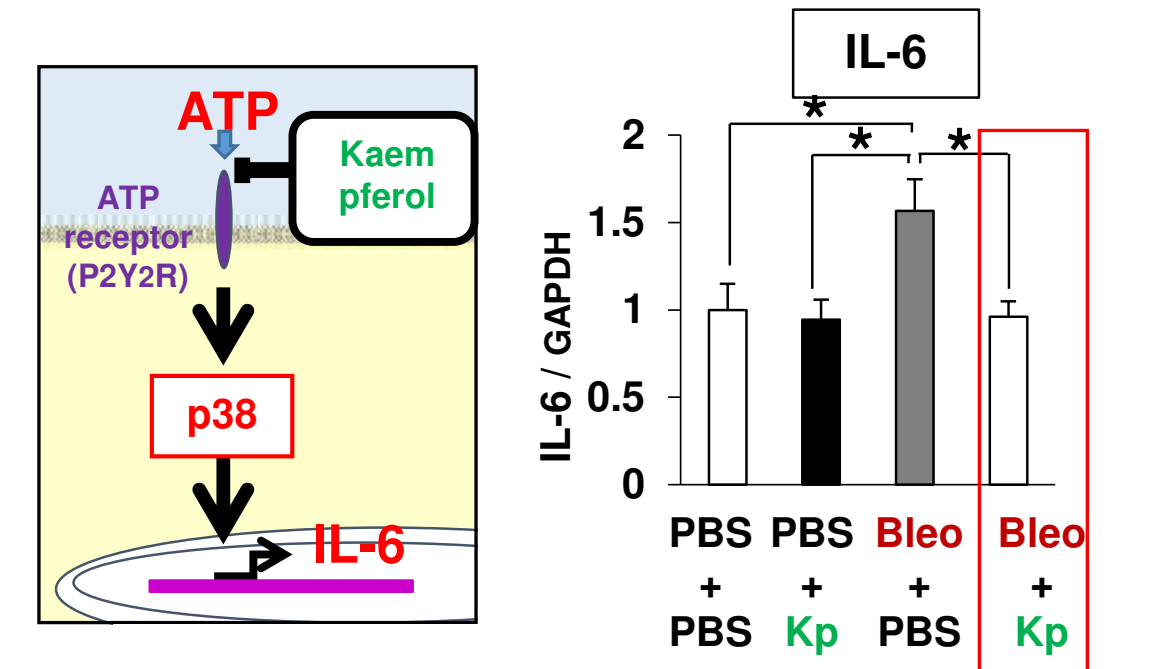

The effect of kaempferol on ATP-induced IL- 6 production and phosphorylation of $\mathrm{p} 38$ in vitro

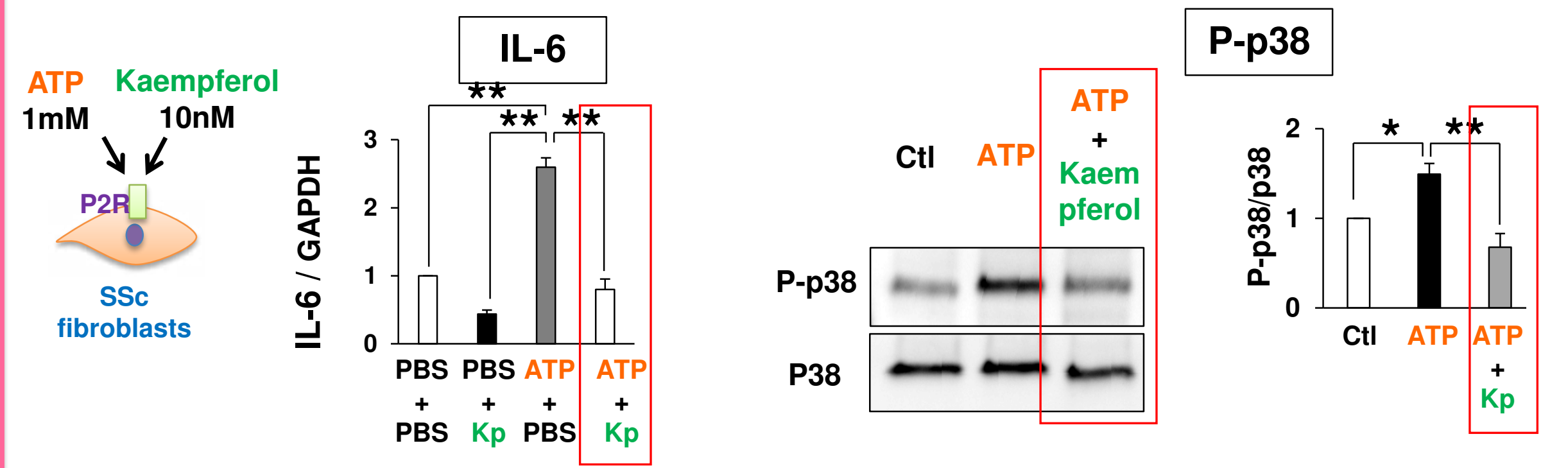

In SSc fibroblast, IL-6 mRNA levels were increased by ATP and Kempferol

- The phosphorylation of $\mathrm{p} 38$ were decreased by Kaempferol.

Kaempferol might inhibited ATP-induced IL-6 production mediated via $\mathrm{P}_{2} \mathrm{Y}_{2}$ receptor.

The model for the regulation of bleomycininduced skin fibrosis by Kaempferol

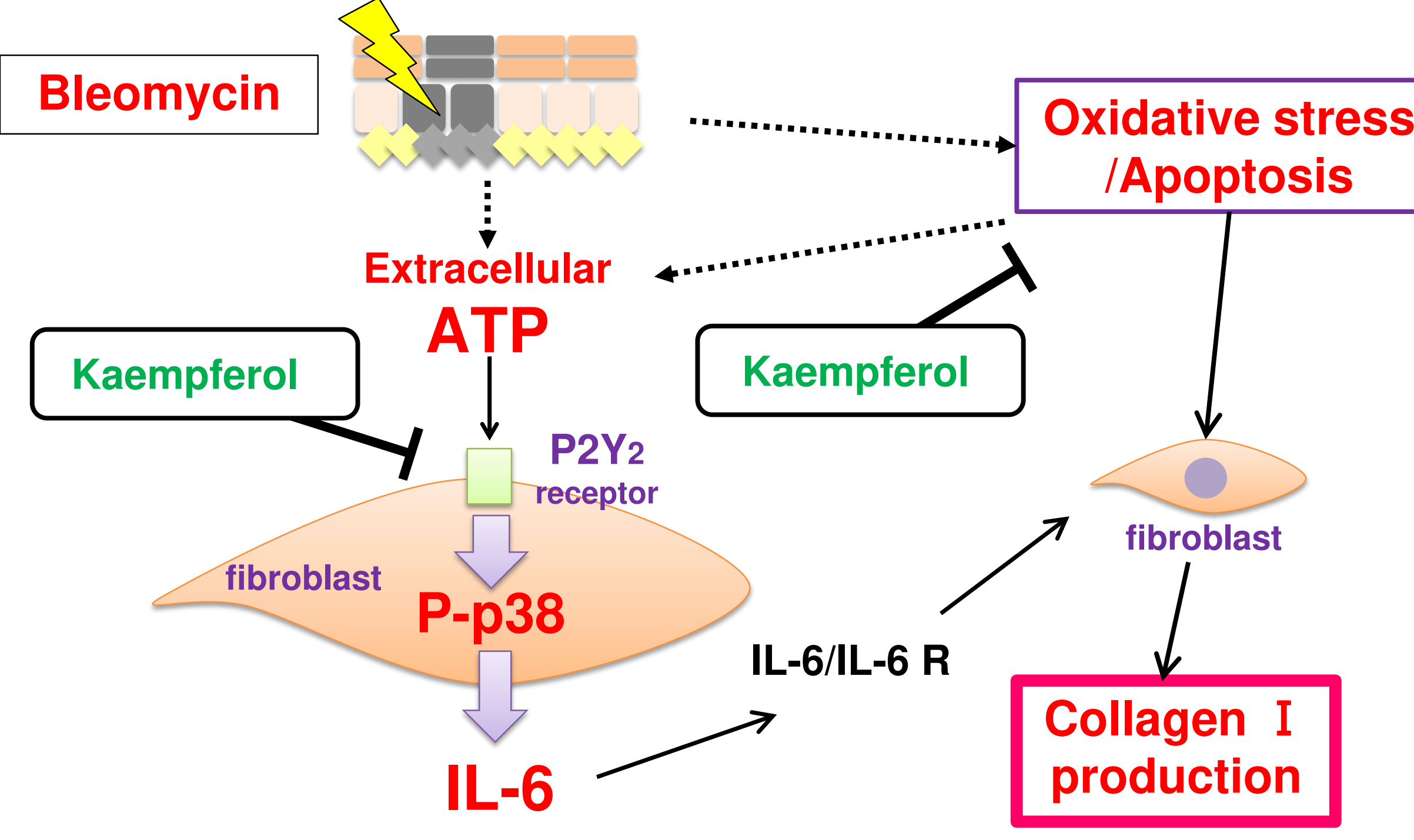

Oxidative stress induced by bleomycin injection increases collagen production from fibroblast.

- Bleomycin also induces extracellular ATP, and IL-6 is induced by ATP mediated by P2Y2 receptor and activation of p38 signaling. IL-6 induces collagen I production from fibroblast.

Kaempferol reduces oxidative stress and inhibits collagen production. Kaempferol also inhibits ATP-induced IL-6 production, leading to decrease

\section{Conclusion}

Kaempferol might improve bleomycin-induced fibrosis by reducing oxidative stress, inflammation, and oxidative cellular damage.

Kaempferol can be an alternative treatment for skin sclerosis in patients with SSc. 